TRANSACTIONS OF THE

AMERICAN MATHEMATICAL SOCIETY

Volume 357, Number 9, Pages 3585-3603

S 0002-9947(04)03595-0

Article electronically published on October 28, 2004

\title{
COMPARING CASTELNUOVO-MUMFORD REGULARITY AND EXTENDED DEGREE: THE BORDERLINE CASES
}

\author{
UWE NAGEL
}

\begin{abstract}
Castelnuovo-Mumford regularity and any extended degree function can be thought of as complexity measures for the structure of finitely generated graded modules. A recent result of Doering, Gunston, and Vasconcelos shows that both can be compared in the case of a graded algebra. We extend this result to modules and analyze when the estimate is in fact an equality. A complete classification is obtained if we choose as extended degree the homological or the smallest extended degree. The corresponding algebras are characterized in three ways: by relations among the algebra generators, by using generic initial ideals, and by their Hilbert series.
\end{abstract}

\section{INTRODUCTION}

In [16] Vasconcelos introduced the concept of an extended degree $\operatorname{Deg}(M)$ of a finitely generated graded module $M$ over the polynomial ring $R=K\left[x_{0}, \ldots, x_{n}\right]$ where $K$ is an infinite field. It is designed to serve as a measure of the size and complexity of the module. Another complexity measure that has attracted a lot of attention is the Castelnuovo-Mumford regularity $\operatorname{reg}(M)$. In [5], Theorem 2.4, it has been shown that both measures can be compared in the case of a standard graded $K$-algebra $A$. In fact, for every extended degree Deg one has

$$
\operatorname{reg} A \leq \operatorname{Deg} A-1 \text {. }
$$

In this note we consider the natural questions of how good this bound is and how it can be extended to finitely generated graded $R$-modules.

Note that a degree shift changes the regularity of the module while its extended degree remains the same. Thus, any comparison between both invariants has to take the degrees of the minimal generators of $M$ into account. Denote by $e_{R}^{+}(M)$ the maximum of these degrees. Then we show as the sought extension to modules in Theorem 3.1 that

$$
\operatorname{reg} M \leq e_{R}^{+}(M)+\operatorname{Deg} M-1 .
$$

Since a standard graded algebra $A$ is (as $R$-module) generated in degree zero this specializes to the earlier result (1.1) in [5].

The key for obtaining estimate (1.2) is the observation that for any extended degree the difference Deg - reg behaves nicely with respect to taking general hyperplane sections (cf. Theorem 2.5) .

Received by the editors April 2, 2003 and, in revised form, December 5, 2003

2000 Mathematics Subject Classification. Primary 13D40, 13D45; Secondary 13P10, 14M05.

Key words and phrases. Castelnuovo-Mumford regularity, extended degree, generic initial ideal, Hilbert series, homological degree, smallest extended degree.

(C)2004 American Mathematical Society 
The bulk of this note is devoted to an analysis of when equality holds in estimate (1.2). Note that if $n \geq 2$ there are many extended degree functions. Thus, we proceed in two steps. First we derive necessary conditions. This is done for an arbitrary extended degree in Section 3, It turns out that equality in estimate (1.2) forces the module to be cyclic. Thus, we are reduced to analyze equality in estimate (1.1). Theorem 3.5 gives a short list of those algebras for which the latter equality has a chance to be true. By specifying an extended degree this result allows us to obtain sufficient conditions for having equality as well.

This is carried out in two cases. The first is Vasconcelos' homological degree hdeg ([16]) which is the prototype of an extended degree. In Corollary 3.7 we characterize the $K$-algebras satisfying $\operatorname{reg}(A)=\operatorname{hdeg}(A)-1$. They form a proper sublist of the algebras described in Theorem 3.5. However, we can hope for a more complete converse of Theorem 3.5 if we choose the extended degree as small as possible. This is considered in Section 4 The existence of a smallest extended degree, denoted by bdeg, has essentially been established by Gunston [8] (cf. also Lemma 4.2). It also has the remarkable property that it does not change when passing to the generic initial module (Lemma 4.3). The main result of this paper characterizes the graded $K$-algebras for which equality holds in estimate (1.1) in case Deg is chosen to be minimal, i.e. we have $\operatorname{reg}(A)=\operatorname{bdeg}(A)-1$. This is true if and only if the algebra is on the list stated in Theorem 3.5 Furthermore, we show that these algebras can also be characterized by using generic initial ideals or by their Hilbert series (cf. Theorems 4.4 and 4.6). It is rather remarkable that in this case purely numerical conditions are equivalent to structural properties.

Another application of Theorem 2.5 is discussed in Section [5. We compare the regularity of the modules $M$ and $M / \mathfrak{q} M$ for certain parameter ideals $\mathfrak{q}$ involving, in particular, the multiplicity of $\mathfrak{q}$ (cf. Proposition 5.2). This broadly generalizes the main result of [14] for generalized Cohen-Macaulay algebras to arbitrary modules.

\section{Comparison With hyperplane SECTION}

The goal of this section is to show a basic inequality about the difference between any extended degree and the Castelnuovo-Mumford regularity. Its consequences will be drawn in the following sections.

We begin by introducing some notation and establishing some preliminary results.

Let $R=K\left[x_{0}, \ldots, x_{n}\right]$ be the polynomial ring over the field $K$ with the standard grading. An extended degree is a numerical function $\mathrm{Deg}: \underline{\bmod }-R \rightarrow \mathbb{Z}$ on the category of finitely generated graded $R$-modules satisfying the following conditions:

(i) If $L=\Gamma_{\mathfrak{m}}(M)$ is the submodule of elements of $M$ annihilated by a power of the homogeneous maximal ideal $\mathfrak{m}$ of $R$, then

$$
\operatorname{Deg} M=\operatorname{Deg} M / L+\ell(L)
$$

where $\ell(-)$ is the length function.

(ii) If $l \in R$ is a sufficiently general regular hyperplane section of $M$, then

$$
\operatorname{Deg} M \geq \operatorname{Deg} M / l M \text {. }
$$

(iii) If $M$ is a Cohen-Macaulay module, then

$$
\operatorname{Deg} M=\operatorname{deg} M,
$$

where $\operatorname{deg}\left(\_\right)$is the ordinary multiplicity function. 
The first example of an extended degree has been the homological degree hdeg introduced by Vasconcelos. The homological degree of the module $M$ is ([16], Definition 2.8)

$$
\operatorname{hdeg} M=\operatorname{deg} M+\sum_{i=n+2-d}^{n+1}\left(\begin{array}{c}
d-1 \\
i-n-2+d
\end{array}\right) \operatorname{hdeg}\left(\operatorname{Ext}_{R}^{i}(M, R)\right),
$$

where $d=\operatorname{dim} M$. Note that the homological degree is defined recursively on the dimension of the module.

The Hilbert function of a module $M \in \underline{\bmod }-R$ is

$$
h_{M}: \mathbb{Z} \rightarrow \mathbb{Z}, h_{M}(j)=\operatorname{rank}_{K}[M]_{j} .
$$

It becomes a polynomial in $j$ for $j \gg 0$. In case $d:=\operatorname{dim} M>0$ this Hilbert polynomial has the form

$$
p_{M}(j)=\frac{\operatorname{deg} M}{(d-1) !} j^{d-1}+\text { lower order terms. }
$$

If $\operatorname{dim} M=0$ we set $\operatorname{deg} M=\ell(M)$.

The Hilbert series of $M$ is just the formal power series

$$
H_{M}(z):=\sum_{j=0}^{\infty} h_{M}(j) \cdot z^{j}
$$

Recall that the irrelevant maximal ideal $\left(x_{0}, \ldots, x_{n}\right)$ of $R$ is denoted by $\mathfrak{m}$. The local cohomology functors $H_{\mathfrak{m}}^{i}(-)$ are the right-derived functors of $\Gamma_{\mathfrak{m}}(-)$.

Let $N$ be any graded $R$-module. Then the end of $N$ is

$$
e(N):=\sup \left\{j \in \mathbb{Z} \mid[N]_{j} \neq 0\right\}
$$

while its initial degree is

$$
a(N)=\inf \left\{j \in \mathbb{Z} \mid[N]_{j} \neq 0\right\} .
$$

Note that $e(N)=-\infty$ if $N$ is trivial. The maximal degree of a minimal generator of a finitely generated module $N$ is denoted by $e_{R}^{+}(N)$, i.e.

$$
e_{R}^{+}(N)=e(N / \mathfrak{m} N) \text {. }
$$

Following [1] we define for $k \geq 0$ integers

$$
r_{k}(M)=\max \left\{i+e\left(H_{\mathfrak{m}}^{i}(M)\right) \mid i \geq k\right\} .
$$

Then $\operatorname{reg} M:=r_{0}(M)$ is the Castelnuovo-Mumford regularity of $M$. The number $r_{1}(M)$ has been called geometric regularity of $M$ in 13 . In order to get an upper bound for $r_{k}(M)$ it suffices to check finitely many conditions. Indeed, according to [11], Theorem 1, we have for $k \geq 1$ that

$$
r_{k}(M) \leq m \quad \text { if and only if }\left[H_{\mathfrak{m}}^{i}(M)\right]_{m+1-i}=0 \text { for all } i \geq k .
$$

This result can be extended to the case $k=0$ as follows.

Lemma 2.1. For any integer $m$ we have

$\operatorname{reg} M \leq m \quad$ if and only if $\quad\left[H_{\mathfrak{m}}^{i}(M)\right]_{m+1-i}=0$ for all $i \geq 0$ and $e_{R}^{+}(M) \leq m$.

Proof. Since $e_{R}^{+}(M) \leq \operatorname{reg} M$ the conditions are necessary. In order to show the other implication we induct on the dimension of $M$. If $\operatorname{dim} M=0$ we have $M \cong$ $H_{\mathfrak{m}}^{0}(M)$. Thus, the assumptions imply $e\left(H_{\mathfrak{m}}^{0}(M)\right) \leq m$. Now let $\operatorname{dim} M \geq 1$. Then we conclude as in the proof of [11], Theorem 1, for $r_{k}$ in case $k \geq 1$. 
Remark 2.2. If $M$ is a graded $K$-algebra $A=R / I$, then the assumption on its generators can be dropped because $e_{R}^{+}(A)=0 \leq \operatorname{reg} A$. In this sense, Lemma 2.1 extends [13, Corollary 1.2, from algebras to modules.

Recall that a polynomial $f \in R$ is called $M$-filter regular if

$$
f \notin \mathfrak{p} \quad \text { for all } \mathfrak{p} \in \text { Ass } M \backslash\{\mathfrak{m}\} .
$$

This is equivalent to the condition that the module $0:_{M} f$ has finite length.

Remark 2.3. Let $l \in R$ be a linear $M$-filter regular element. Then it follows that

$$
H_{\mathfrak{m}}^{i}\left(M / 0:_{M} l\right) \cong H_{\mathfrak{m}}^{i}(M) \text { for all } i \geq 1 .
$$

Hence, the exact sequence induced by multiplication

$$
0 \rightarrow\left(M / 0:_{M} l\right)(-1) \rightarrow M \rightarrow M / l M \rightarrow 0
$$

provides the exact sequence

$$
\begin{aligned}
& 0 \longrightarrow\left(0:_{M} l\right)(-1) \longrightarrow H_{\mathfrak{m}}^{0}(M)(-1) \stackrel{l}{\longrightarrow} H_{\mathfrak{m}}^{0}(M) \longrightarrow H_{\mathfrak{m}}^{0}(M / l M) \longrightarrow \ldots \\
& \ldots \longrightarrow H_{\mathfrak{m}}^{i}(M) \longrightarrow H_{\mathfrak{m}}^{i}(M / l M) \longrightarrow H_{\mathfrak{m}}^{i+1}(M)(-1) \stackrel{l}{\longrightarrow} H_{\mathfrak{m}}^{i+1}(M) \longrightarrow \ldots
\end{aligned}
$$

It is well known that $\operatorname{reg}(M / l M) \leq \operatorname{reg}(M)$ if $l$ is an $M$-filter regular linear form. Thus, the next observation is vacuous if and only if $\operatorname{reg}(M / l M)=\operatorname{reg}(M)$.

Lemma 2.4. Let $l \in R$ be an $M$-filter regular linear form. Then we have

$$
\left[H_{\mathfrak{m}}^{0}(M)\right]_{j} \neq 0 \quad \text { if } \operatorname{reg}(M / l M)+1 \leq j \leq \operatorname{reg}(M) .
$$

Proof. The exact sequence (2.2) implies for the geometric regularity (cf., for example, 11], Lemma 2)

$$
r_{1}(M) \leq \operatorname{reg} M / l M .
$$

Hence, if $r:=\operatorname{reg} M>\operatorname{reg} M / l M$, then we must have $\left[H_{\mathfrak{m}}^{0}(M)\right]_{r} \neq 0$. Now consider the following piece of the sequence (2.2):

$$
\left[H_{\mathfrak{m}}^{0}(M)\right]_{j-1} \rightarrow\left[H_{\mathfrak{m}}^{0}(M)\right]_{j} \rightarrow\left[H_{\mathfrak{m}}^{0}(M / l M)\right]_{j} .
$$

It shows for $j>\operatorname{reg}(M / l M)$ that $\left[H_{\mathfrak{m}}^{0}(M)\right]_{j} \neq 0$ implies $\left[H_{\mathfrak{m}}^{0}(M)\right]_{j-1} \neq 0$. Our claim follows.

As a final piece of notation we set

$$
\left[h_{\mathfrak{m}}^{i}(M)\right]_{j}=\operatorname{rank}_{K}\left[H_{\mathfrak{m}}^{i}(M)\right]_{j} .
$$

Now we are ready for the main result of this section.

Theorem 2.5. Let $M$ be a finitely generated graded $R$-module of positive dimension. Suppose $K$ is an infinite field. Then we have for every sufficiently general linear form $l \in R$ and any extended degree Deg

$$
\operatorname{Deg}(M / l M)-\operatorname{reg}(M / l M) \leq \operatorname{Deg}(M)-\operatorname{reg}(M) .
$$

Proof. Since $K$ is infinite, $l$ is $M$-filter regular.

Let $N$ be any $R$-module. Following [9] we call $N^{s m}:=N / H_{\mathfrak{m}}^{0}(N)$ the slight modification of $N$. If it is non-trivial, then it has positive depth.

For short put $N=M^{s m}$. Comparing the sequences (2.2) for $M$ and $N$ we obtain the exact sequence

$$
0 \longrightarrow H_{\mathfrak{m}}^{0}(M) / l H_{\mathfrak{m}}^{0}(M) \longrightarrow H_{\mathfrak{m}}^{0}(M / l M) \longrightarrow H_{\mathfrak{m}}^{0}(N / l N) \longrightarrow 0
$$


Next, consider the commutative diagram where the vertical maps are multiplication by $l$

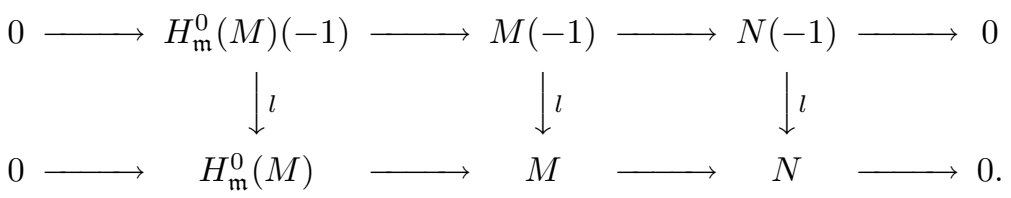

The Snake lemma provides the exact sequence

$$
0 \longrightarrow H_{\mathfrak{m}}^{0}(M) / l H_{\mathfrak{m}}^{0}(M) \longrightarrow M / l M \longrightarrow N / l N \longrightarrow 0 .
$$

Its long exact cohomology sequence induces isomorphisms

$$
H_{\mathfrak{m}}^{i}(M / l M) \cong H_{\mathfrak{m}}^{i}(N / l N) \quad \text { for all } i \geq 1 .
$$

Moreover, the sequence (2.3) implies $e\left(H_{\mathfrak{m}}^{0}(M / l M)\right) \geq e\left(H_{\mathfrak{m}}^{0}(N / l N)\right)$. We conclude that

$$
\operatorname{reg}(N / l N) \leq \operatorname{reg}(M / l M) .
$$

Comparing the sequences (2.3) and (2.4) we obtain a graded isomorphism

$$
(M / l M)^{s m} \cong(N / l N)^{s m} .
$$

Hence, conditions (i) and (ii) for the extended degree imply

$$
\begin{aligned}
\operatorname{Deg}(M / l M) & =\ell\left(H_{\mathfrak{m}}^{0}(M / l M)\right)+\operatorname{Deg}\left((M / l M)^{s m}\right) \\
& =\ell\left(H_{\mathfrak{m}}^{0}(M / l M)\right)+\operatorname{Deg}\left((N / l N)^{s m}\right) \\
& \leq \sum_{j \leq \operatorname{reg}(M / l M)}\left(\left[h_{\mathfrak{m}}^{0}(M)\right]_{j}+\left[h_{\mathfrak{m}}^{0}(N / l N)\right]_{j}\right)+\operatorname{Deg}\left((N / l N)^{s m}\right) \\
& =\sum_{j \leq \operatorname{reg}(M / l M)}\left[h_{\mathfrak{m}}^{0}(M)\right]_{j}+\operatorname{Deg}(N / l N) \\
& \leq \sum_{j \leq \operatorname{reg}(M / l M)}\left[h_{\mathfrak{m}}^{0}(M)\right]_{j}+\operatorname{Deg}(N),
\end{aligned}
$$

where the first estimate is a consequence of sequence (2.3) and $\operatorname{reg}(N / l N) \leq$ $\operatorname{reg}(M / l M)$ is used to justify the equality following it. Note that $\operatorname{depth} N>0$ allows us to conclude $\operatorname{Deg}(N / l N) \leq \operatorname{Deg}(N)$ by condition (ii) for the extended degree.

Now, Lemma 2.4 provides the trivial estimate

$$
\operatorname{reg}(M)-\operatorname{reg}(M / l M) \leq \sum_{j=\operatorname{reg}(M / l M)+1}^{\operatorname{reg}(M)}\left[h_{\mathfrak{m}}^{0}(M)\right]_{j} .
$$

Summing up we obtain

$$
\operatorname{reg}(M)-\operatorname{reg}(M / l M)+\operatorname{Deg}(M / l M) \leq \ell\left(H_{\mathfrak{m}}^{0}(M)\right)+\operatorname{Deg}(N)=\operatorname{Deg}(M),
$$

which proves the claim. 
For later use we record when we have equality in Theorem 2.5.

Corollary 2.6. Adopting the notation and assumptions of Theorem 2.5 we have

$$
\operatorname{Deg}(M / l M)-\operatorname{reg}(M / l M)=\operatorname{Deg}(M)-\operatorname{reg}(M)
$$

if and only if the following conditions are satisfied:

$$
\begin{aligned}
& {\left[H_{\mathfrak{m}}^{0}(M)\right]_{j}=\left[0:_{M} l\right]_{j} \quad \text { for all } j<\operatorname{reg}(M / l M),} \\
& {\left[h_{\mathfrak{m}}^{0}(M)\right]_{j}=1 \quad \text { if } \operatorname{reg}(M / l M)+1 \leq j \leq \operatorname{reg}(M),} \\
& \operatorname{Deg}(N / l N)=\operatorname{Deg}(N) \quad \text { where } N:=M / H_{\mathfrak{m}}^{0}(M) .
\end{aligned}
$$

Proof. Analyzing the estimates in the proof of Theorem 2.5 we see that we have equality in its claim if and only if conditions (II) and (III) are satisfied and there are equations

$$
\left[h_{\mathfrak{m}}^{0}(M / l M)\right]_{j}=\left[h_{\mathfrak{m}}^{0}(M)\right]_{j}+\left[h_{\mathfrak{m}}^{0}(N / l N)\right]_{j} \quad \text { for all } j \leq \operatorname{reg}(M / l M) .
$$

But the sequences (2.2) and (2.3) provide the exact sequence

$$
0 \rightarrow\left(0:_{M} l\right)(-1) \rightarrow H_{\mathfrak{m}}^{0}(M)(-1) \rightarrow H_{\mathfrak{m}}^{0}(M) \rightarrow H_{\mathfrak{m}}^{0}(M / l M) \rightarrow H_{\mathfrak{m}}^{0}(N / l N) \rightarrow 0 .
$$

Therefore (2.5) is equivalent to condition (I).

We now begin to discuss the consequences of Theorem 2.5.

Corollary 2.7. If $\operatorname{Deg}(M / l M)=\operatorname{Deg}(M)$, then $\operatorname{reg}(M / l M)=\operatorname{reg}(M)$.

Proof. Using Theorem 2.5 the assumption implies

$$
\operatorname{reg}(M) \leq \operatorname{reg}(M / l M),
$$

but for a general linear form $l$ we always have $\operatorname{reg}(M) \geq \operatorname{reg}(M / l M)$.

Remark 2.8. So far, the best known extended degree is Vasconcelos' homological degree. An example in [16], Remark 2.15, shows that there are modules having positive depth such that

$$
\operatorname{hdeg}(M / l M)<\operatorname{hdeg}(M) .
$$

Vasconcelos points out that it would be considerably better to have an extended degree where equality holds in condition (II). However, it is asking too much to hope for the existence of an extended degree such that $\operatorname{Deg}(M / l M)=\operatorname{Deg}(M)$ for general $l$ and every module $M$. Indeed, there are plenty of modules such that

$$
\operatorname{reg}(M / l M)<\operatorname{reg}(M) .
$$

\section{Modules With maximal Regularity}

We will establish a bound on the regularity and then investigate the modules whose Castelnuovo-Mumford regularity is maximal with respect to this bound. It turns out that this property puts rather severe restrictions on the module structure.

We will assume that the ground field $K$ is infinite (but confer Remark 5.1).

The following result states the announced regularity bound.

Theorem 3.1. Suppose $K$ is an infinite field. Then we have for every finitely generated, graded R-module

$$
\operatorname{reg}(M) \leq e_{R}^{+}(M)+\operatorname{Deg}(M)-1 .
$$


Proof. Let $l \in R$ be a general linear form. Since $e_{R}^{+}(M)=e_{R}^{+}(M / l M)$, Theorem 2.5 shows that, by induction, it suffices to prove the claim if $\operatorname{dim} M=0$. But in this case we have $\operatorname{reg}(M)=e(M)$ and

$$
[M]_{j} \neq 0 \text { if } e_{R}^{+}(M) \leq j \leq e(M) .
$$

It follows that

and we are done.

$$
e(M)-e_{R}^{+}(M)+1 \leq \ell(M)
$$

If $A=R / I$ is a standard graded $K$-algebra, then $e_{R}^{+}(A)=0$. Therefore, the theorem above has the following consequence which has first been shown as Theorem 2.4 in 5 .

Corollary 3.2. Let $A$ be a standard graded $K$-algebra where $K$ is an infinite field. Then

$$
\operatorname{reg}(A) \leq \operatorname{Deg}(A)-1
$$

The goal of the remainder of this section is to study the modules which have maximal regularity with respect to Theorem 3.1

The next observation shows that it suffices to characterize equality for algebras.

Lemma 3.3. Let $M$ be a finitely generated, graded $R$-module such that

$$
\operatorname{reg}(M)=e_{R}^{+}(M)+\operatorname{Deg}(M)-1
$$

Suppose $K$ is infinite and let $l \in R$ be a general linear form. Then we have:

(a) If $\operatorname{dim} M \geq 1$, then

$$
\operatorname{reg}(M / l M)=e_{R}^{+}(M / l M)+\operatorname{Deg}(M / l M)-1 .
$$

(b) $M$ is a cyclic $R$-module.

Proof. We will twice use the fact that the degrees of the minimal generators of $M$ and of $M / l M$ coincide for general $l$. First, it shows that claim (a) is a consequence of Theorem 3.1 and Theorem 2.5 .

Second, using induction on $\operatorname{dim} M$ and part (a) we see that it suffices to show claim (b) if $\operatorname{dim}(M)=0$. In this case the assumption becomes

$$
e(M)-e_{R}^{+}(M)+1=\ell(M) .
$$

Hence condition (3.1) implies

$$
h_{M}(j)=1 \quad \text { if } a(M)=e_{R}^{+}(M) \leq j \leq e(M)
$$

which in particular proves the claim.

We need some preparation for the next result. The graded $K$ dual of an $R$ module $N$ is denoted by $M^{\vee}=\bigoplus_{j \in \mathbb{Z}} \operatorname{Hom}_{K}\left([M]_{-j}, K\right)$. By local duality

$$
H_{\mathfrak{m}}^{i}(M)^{\vee} \cong \operatorname{Ext}_{R}^{n+1-i}(M, R)(-n-1)
$$

is a finitely generated graded $R$-module.

Lemma 3.4. In $R=K\left[x_{0}, \ldots, x_{n}\right]$ consider the ideal

$$
I=\left(f_{n} l_{0}, f_{n} f_{n-1} l_{1}, \ldots, f_{n} \ldots f_{t+1} l_{n-t-1}, f_{n} \ldots f_{t}\right)
$$

where $0 \leq t \leq n$, every $f_{i} \neq 0$ is a homogenous polynomial of degree $d_{i} \geq 0, d_{n}, d_{t} \geq$ 1 , and every $\bar{l}_{i}$ is a linear form. (Note that in case $n=t$ the ideal $I$ is simply defined 
as $I=\left(f_{n}\right)$.) If I has (as indicated) $n+1-t$ minimal generators, then the local cohomology modules of $A=R / I$ are

$$
H_{\mathfrak{m}}^{i}(A)^{\vee} \cong \begin{cases}\left(R /\left(l_{0}, \ldots, l_{n-1-i}, f_{i}\right)\right)\left(d_{i}+\ldots+d_{n}-i-1\right) & \text { if } t \leq i \leq n, \\ 0 & \text { otherwise. }\end{cases}
$$

In particular, $H_{\mathfrak{m}}^{i}(A)^{\vee}$ either vanishes or is (up to a degree shift) a hypersurface ring of dimension $i$.

Furthermore, the Hilbert series of $A$ is

$$
H_{A}(z)=\sum_{j=t}^{n} \frac{1-z^{d_{j}}}{(1-z)^{j+1}} \prod_{i=j+1}^{n} z^{d_{i}}
$$

where the product is defined to be 1 if $j+1>n$.

Proof. Since $I$ has $n+1-t$ minimal generators we see that

$$
\begin{aligned}
& l_{j} \notin\left(l_{0}, \ldots, l_{j-1}\right) \quad \text { if } 0 \leq j \leq t, \\
& f_{j} \notin\left(l_{0}, \ldots, l_{n-j-1}\right) \quad \text { if } t \leq j \leq n .
\end{aligned}
$$

It follows that the rings $R /\left(l_{0}, \ldots, l_{n-1-i}, f_{i}\right)$ have the asserted dimension $i$ and that $f_{n}$ is the greatest common divisor of the polynomials in $I$. Thus, we can write $I=\left(f_{n}\right) \cap \mathfrak{b}$, where $\mathfrak{b} \subset R$ is an ideal having codimension at least two or $\mathfrak{b}=R$. Consider the exact sequence

$$
0 \rightarrow\left(f_{n}\right) / \mathfrak{b} \cap\left(f_{n}\right) \rightarrow R / I \rightarrow R /\left(f_{n}\right) \rightarrow 0 .
$$

If $I$ is not a principal ideal, then the dimension of $\left(f_{n}\right) / \mathfrak{b} \cap\left(f_{n}\right) \cong\left(\mathfrak{b}+\left(f_{n}\right)\right) / \mathfrak{b}$ is less than $\operatorname{dim} R / I$. Thus, using the long exact cohomology sequence we get in any case

$$
H_{\mathfrak{m}}^{n}(A) \cong H_{\mathfrak{m}}^{n}\left(R / f_{n} R\right) \cong\left(R / f_{n} R\right)^{\vee}\left(-d_{n}+n+1\right)
$$

as claimed. (The first isomorphism is also a consequence of the more general Proposition 3.7 in [4].)

In order to compute the cohomology of $A$ we induct on $n$. If $n=0$, then $I=\left(f_{n}\right)$ and the last computation gives the claim. Let $n \geq 1$. Due to the last isomorphism it suffices to compute the $i$-th cohomology module for $i \leq n-1$.

Multiplication by $f_{n}$ on $A$ induces the exact sequence

$$
0 \rightarrow\left(R / I: f_{n}\right)\left(-d_{n}\right) \rightarrow R / I \rightarrow R / f_{n} R \rightarrow 0 .
$$

Since $R / f_{n} R$ is Cohen-Macaulay of dimension $n$ it provides isomorphisms

$$
H_{\mathfrak{m}}^{i}(A) \cong H_{\mathfrak{m}}^{i}\left(R / I: f_{n}\right)\left(-d_{n}\right) \quad(i \leq n-1) .
$$

Consider the following ideal in $R$ :

$$
J=\left(f_{n-1} l_{1}, \ldots, f_{n-1} \ldots f_{t+1} l_{n-t-1}, f_{n-1} \ldots f_{t}\right) .
$$

We have

$$
I: f_{n}=l_{0} R+J .
$$

Thus, putting $\bar{R}=R / l_{0} R$ we get the isomorphism

$$
R / I: f_{n} \cong \bar{R} / J \bar{R} .
$$

But the induction hypothesis applies to $J \bar{R}$. Hence, the isomorphisms (3.4) provide the claim about the cohomology of $A$. 
It remains to determine the Hilbert series of $A$. If $n=0$ we obtain

$$
H_{A}(z)=\sum_{j=0}^{d_{0}-1} z^{j}=\frac{1-z^{d_{0}}}{1-z}
$$

as claimed. Let $n \geq 1$. If $t=n$, then $A$ is a hypersurface ring of degree $d_{n}$ and its Hilbert series is $H_{A}(z)=\frac{1-z^{d_{n}}}{(1-z)^{n+1}}$. Let $t<n$. Then the sequence (3.3) and the isomorphism (3.5) together with the induction hypothesis provide

$$
\begin{aligned}
H_{A}(z) & =\frac{1-z^{d_{n}}}{(1-z)^{n+1}}+z^{d_{n}}\left[\sum_{j=t}^{n-1} \frac{1-z^{d_{j}}}{(1-z)^{j+1}} \prod_{i=j+1}^{n-1} z^{d_{i}}\right] \\
& =\sum_{j=t}^{n} \frac{1-z^{d_{j}}}{(1-z)^{j+1}} \prod_{i=j+1}^{n} z^{d_{i}},
\end{aligned}
$$

completing the proof.

We are ready for the main result of this section.

Theorem 3.5. Let $A \neq 0$ be a standard graded $K$-algebra where $K$ is an infinite field. If

$$
\operatorname{reg}(A)=\operatorname{Deg}(A)-1
$$

for some extended degree Deg, then $A$ is (as $K$-algebra) isomorphic to $R / I$, where $R=K\left[x_{0}, \ldots, x_{n}\right], n=\operatorname{dim} A$, and

$$
I=\left(f_{n} x_{0}, f_{n} f_{n-1} x_{1}, \ldots, f_{n} \ldots f_{t+1} x_{n-t-1}, f_{n} \ldots f_{t}\right)
$$

with homogenous polynomials $f_{i} \neq 0$ of degree $d_{i} \geq 0, t=\operatorname{depth} A$, and $d_{n}, d_{t} \geq 1$ such that $f_{i} \notin\left(x_{0}, \ldots, x_{n-i-1}\right)$ for all $i=t, \ldots, n-1$.

Proof. We will proceed in several steps. First, we show several properties of the algebras in question, second, we use induction to conclude the proof.

Step 1. Assume $\operatorname{dim} A=0$. Then we will show that $A \cong K[x] / x^{d}$ for some integer $d \geq 0$.

Indeed, (3.2) reads in this case as

$$
h_{A}(j)=1 \text { if } 0 \leq j \leq e(A) .
$$

In particular, $\operatorname{rank}_{K}[A]_{1} \leq 1$ implies that $A$ is isomorphic to a quotient of a polynomial ring in one variable. The claim of Step 1 follows.

Step 2. Put $B=A / H_{\mathfrak{m}}^{0}(A)$. Then we claim that

$$
\operatorname{reg}(B) \leq \operatorname{reg}(A)-\ell\left(H_{\mathfrak{m}}^{0}(A)\right) .
$$

This assertion is trivially true if $\operatorname{depth} A>0$. Thus, assume $H_{\mathfrak{m}}^{0}(A) \neq 0$. Then the assumption and Theorem 3.1 provide

$$
\operatorname{reg}(A)+1=\operatorname{Deg}(A)=\operatorname{Deg}(B)+\ell\left(H_{\mathfrak{m}}^{0}(A)\right) \geq \operatorname{reg}(B)+1+\ell\left(H_{\mathfrak{m}}^{0}(A)\right)
$$

and inequality (3.6) is shown.

Step 3. Suppose $\operatorname{dim} A \geq 1$ and $H_{\mathfrak{m}}^{0}(A) \neq 0$. Then we show that

$$
\ell\left(H_{\mathfrak{m}}^{0}(A)\right) \leq e\left(H_{\mathfrak{m}}^{0}(A)\right)-a\left(H_{\mathfrak{m}}^{0}(A)\right)+1 .
$$

In order to do that write $A=R / I$ and denote the saturation of $I$ by $I^{\text {sat }}$. There are isomorphisms $H_{\mathfrak{m}}^{0}(A) \cong I^{\text {sat }} / I$ and $B \cong R / I^{\text {sat }}$. Estimate (3.6) provides 
$\operatorname{reg}(A)>\operatorname{reg}(B)=r_{1}(A)$ which implies $\operatorname{reg}(A)=e\left(H_{\mathfrak{m}}^{0}(A)\right)$. Since $\operatorname{reg}\left(I^{\text {sat }}\right)=$ $\operatorname{reg}(B)+1$ it follows using estimate (3.6) again that

$$
\left\{\begin{aligned}
a\left(H_{\mathfrak{m}}^{0}(A)\right) \leq e_{R}^{+}\left(I^{s a t}\right) & \leq \operatorname{reg}(B)+1, \\
& \leq \operatorname{reg}(A)+1-\ell\left(H_{\mathfrak{m}}^{0}(A)\right), \\
& =e\left(H_{\mathfrak{m}}^{0}(A)\right)+1-\ell\left(H_{\mathfrak{m}}^{0}(A)\right)
\end{aligned}\right.
$$

which gives our claimed estimate (3.8).

Step 4 . If $H_{\mathfrak{m}}^{0}(A)$ is non-trivial, then we claim that it is a cyclic $R$-module and

$$
\left[h_{\mathfrak{m}}^{0}(A)\right]_{j}=1 \quad \text { if } a\left(H_{\mathfrak{m}}^{0}(A)\right) \leq j \leq e\left(H_{\mathfrak{m}}^{0}(A)\right) .
$$

We argue by induction on $\operatorname{dim} A$. If $\operatorname{dim} A=0$ this claim is shown in Step 1. Let $\operatorname{dim} A>0$ and let $l$ be a general linear form. Then the sequence (2.2) provides an embedding

$$
H_{\mathfrak{m}}^{0}(A) / l H_{\mathfrak{m}}^{0}(A) \hookrightarrow H_{\mathfrak{m}}^{0}(A / l A) .
$$

According to Lemma [3.3, (a), the induction hypothesis applies to $A / l A$. Hence $H_{\mathfrak{m}}^{0}(A) / l H_{\mathfrak{m}}^{0}(A)$ and thus $H_{\mathfrak{m}}^{0}(A)$ must be a cyclic module. This implies

$$
e\left(H_{\mathfrak{m}}^{0}(A)\right)-a\left(H_{\mathfrak{m}}^{0}(A)\right)+1 \leq \ell\left(H_{\mathfrak{m}}^{0}(A)\right) .
$$

Comparing with estimate (3.8) we get

$$
e\left(H_{\mathfrak{m}}^{0}(A)\right)-a\left(H_{\mathfrak{m}}^{0}(A)\right)+1=\ell\left(H_{\mathfrak{m}}^{0}(A)\right)
$$

which implies the claim of this step about the Hilbert function of $H_{\mathfrak{m}}^{0}(A)$.

Step 5. Now we can show

(i) If $H_{\mathfrak{m}}^{0}(A) \neq 0$, then $a\left(H_{\mathfrak{m}}^{0}(A)\right)=e_{R}^{+}\left(I^{\text {sat }}\right)=r_{1}(A)+1$.

(ii) If $\operatorname{dim} A>0$, then $\operatorname{reg}(B)=\operatorname{Deg}(B)-1$.

Indeed, (3.10) implies equality for all the estimates in (3.9). This shows claim (i) and

Since by assumption

$$
\operatorname{reg}(B)=\operatorname{reg}(A)-\ell\left(H_{\mathfrak{m}}^{0}(A)\right) .
$$

$$
\operatorname{reg}(A)=\operatorname{Deg}(A)-1=\operatorname{Deg}(B)+\ell\left(H_{\mathfrak{m}}^{0}(A)\right)-1
$$

claim (ii) follows.

Step 6. Suppose temporarily that $H_{\mathfrak{m}}^{0}(A) \cong I^{\text {sat }} / I$ is non-trivial. Then claim (i) in Step 5 says that there is a minimal generator of $I^{\text {sat }}$, say $f$, of maximal degree whose residue class generates $H_{\mathfrak{m}}^{0}(A)$ as $R$-module. Combined with Step 4 we conclude that in any case there is a homogeneous ideal $\mathfrak{a}$ such that

$$
I^{s a t}=\mathfrak{a}+f R \quad \text { and } \quad I=\mathfrak{a}+f\left(l_{0}^{k}, l_{1}, \ldots, l_{n}\right)
$$

for some integer $k \geq 0$ where $\left\{l_{0}, l_{1}, \ldots, l_{n}\right\}$ is a regular sequence of linear forms. (Note that $I=I^{\text {sat }}$ if and only if $k=0$.)

Step 7. Now we are ready to establish the assertion of Theorem 3.5. We will use Step 6 and argue by induction on $\operatorname{dim} A$. If $\operatorname{dim} A=0$ the claim is shown in Step 1. Let $\operatorname{dim} A>0$. Write $A=R / I$. First, suppose that $\operatorname{depth} A>0$. Let $l$ be a general linear form. After a change of coordinates we may assume $l=x_{n}$. Lemma 3.3 shows that the induction hypothesis applies to $A / l A \cong \bar{R} / \bar{I}$ where $\bar{R}=R / x_{n} R$. Since $l$ is not a zero divisor for $A$ the minimal generators of $\bar{R}$ lift to minimal generators of $I$ preserving their factorization. The claim of our statement follows. (Note that $\operatorname{depth} A / l A=\operatorname{depth} A-1$.) 
Second, suppose $\operatorname{depth} A=0$. Then $t:=\operatorname{depth} R / I^{\text {sat }} \geq 1$. Hence, by Step 5 , (ii) and the first part of Step 7 we may assume that $I^{\text {sat }}$ is of the form as claimed. In particular, $f_{n} \cdots f_{t}$ is the unique minimal generator of largest degree of $I^{\text {sat }}$ if $\operatorname{deg} f_{t} \geq 2$. If $\operatorname{deg} f_{t}=1$ we may still assume that we have in Step $6 f=f_{n} \ldots f_{t}$ and

$$
\mathfrak{a}=\left(f_{n} x_{0}, f_{n} f_{n-1} x_{1}, \ldots, f_{n} \ldots f_{t+1} x_{n-t-1}\right)
$$

such that $I=\mathfrak{a}+f\left(f_{0}, l_{1}, \ldots, l_{n}\right)$ with suitable linear forms $l_{i}$ and a form $f_{0} \neq 0$ of degree $d_{0} \geq 1$. Since $f\left(x_{0}, \ldots, x_{n-1-t}\right) \subset \mathfrak{a}$ we get

$$
\left(x_{0}, \ldots, x_{n-1-t}\right) \subset \operatorname{Ann} H_{\mathfrak{m}}^{0}(A)=\left(f_{0}, l_{1}, \ldots, l_{n}\right) .
$$

Thus, possibly after a change of coordinates we may assume

$$
\left(f_{0}, l_{1}, \ldots, l_{n}\right)=\left(x_{0}, \ldots, x_{n-1}, f_{0}\right) .
$$

Then $I=\mathfrak{a}+f\left(x_{0}, \ldots, x_{n-1}, f_{0}\right)$ is of the form as claimed.

Combined with Lemma 3.3 we obtain

Corollary 3.6. Suppose the ground field $K$ is infinite. Let $M \neq 0$ be a finitely generated graded $R$-module such that

$$
\operatorname{reg}(M)=e_{R}^{+}(M)+\operatorname{Deg}(M)-1
$$

for some extended degree Deg. Then $M$ is a cyclic $R$-module which is up to a degree shift as graded $K$-algebra isomorphic to one of the algebras in Theorem 3.5 .

Using as extended degree Vasconcelos' homological degree (cf. (2.1) ) we get a complete characterization.

Corollary 3.7. Let $K$ be an infinite field and let $M \neq 0$ be a finitely generated graded $R$-module such that

$$
\operatorname{reg}(M)=e_{R}^{+}(M)+\operatorname{hdeg}(M)-1 .
$$

Then $M$ is a cyclic $R$-module which is up to a degree shift as graded $K$-algebra isomorphic to $R / I$, where

$$
I=\left(f_{n} x_{0}, f_{n} f_{n-1}\left(x_{1}, \ldots, x_{n-1}, f_{0}\right)\right)
$$

with homogeneous polynomials $f_{i} \neq 0$ of degree $d_{i} \geq 0, d_{n} \geq 1, f_{n-1} \notin\left(x_{0}\right)$, and $f_{0} \notin\left(x_{0}, \ldots, x_{n-1}\right)$.

Proof. According to the previous result it suffices to compare the regularity and the homological degree of the algebras in Theorem 3.5. Let $A$ be such an algebra. Put $t=\operatorname{depth} A$. Note that $\operatorname{dim} A=n$. Then Lemma 3.4 provides

$$
\operatorname{reg}(A)=d_{t}+\ldots+d_{n}-1
$$

and

$$
\operatorname{hdeg}(A)=d_{n}+\sum_{i=t}^{n-1}\left(\begin{array}{c}
n-1 \\
i
\end{array}\right) d_{i} .
$$

Therefore, we get $\operatorname{reg}(A)=\operatorname{hdeg}(A)-1$ if and only if $d_{i}=0$ for all $i$ such that $1 \leq i \leq n-2$ proving the claim.

In the next section we will show that there is an extended degree function bdeg such that we have for every algebra $A$ as in Theorem 3.5

$$
\operatorname{reg}(A)=\operatorname{bdeg}(A)-1\left(=d_{t}+\ldots+d_{n}-1\right) .
$$




\section{The SMAllest EXTEnded DEgReE}

In his thesis [8] T. Gunston introduced a very particular extended degree which can be described as the smallest among all extended degrees. Using this degree we obtain several characterizations of the algebras occurring in Theorem 3.5 .

Let us begin with the axiomatic description of this degree (cf. [8], Theorem 3.1.2).

Theorem 4.1. Let $K$ be an infinite field. Then there is a unique numerical function bdeg $: \underline{\bmod }-R \rightarrow \mathbb{Z}$ satisfying the following conditions:

(1) If $L=\Gamma_{\mathfrak{m}}(M)$, then

$$
\operatorname{bdeg}(M)=\operatorname{bdeg}(M / L)+\ell(L) .
$$

(2) If $l \in R$ is a sufficiently general regular hyperplane section of $M$, then

$$
\text { bdeg } M=\operatorname{bdeg} M / l M \text {. }
$$

(3) $\operatorname{bdeg}(0)=0$.

T. Gunston called the function bdeg the extremal cohomological degree. It is easy to see that it is an extended degree and in this note we prefer to call the function the smallest extended degree. This is justified because of the next observation.

Lemma 4.2. If the field $K$ is infinite, then we have for every extended degree Deg on $\underline{\bmod }-R$

$$
\operatorname{bdeg}(M) \leq \operatorname{Deg}(M) \quad \text { for all } M \in \underline{\bmod }-R \text {. }
$$

Proof. The result is contained in T. Gunston's thesis [8]. However, for the convenience of the reader we include the short argument using the axiomatic description only.

We induct on $\operatorname{dim} M$. If $\operatorname{dim} M=0$, then $M$ has finite length and

$$
\operatorname{bdeg}(M)=\operatorname{Deg}(M)=\ell(M) .
$$

Let $\operatorname{dim} M>0$. Then $N:=M / H_{\mathfrak{m}}^{0}(M)$ has positive depth and by induction we have for a general linear form $l \in R$ that $\operatorname{bdeg}(N / l N) \leq \operatorname{Deg}(N / l N)$. Thus, it follows that

$$
\begin{aligned}
\operatorname{bdeg}(M)=\operatorname{bdeg}(N)+\ell\left(H_{\mathfrak{m}}^{0}(M)\right) & =\operatorname{bdeg}(N / l N)+\ell\left(H_{\mathfrak{m}}^{0}(M)\right) \\
& \leq \operatorname{Deg}(N / l N)+\ell\left(H_{\mathfrak{m}}^{0}(M)\right) \\
& \leq \operatorname{Deg}(N)+\ell\left(H_{\mathfrak{m}}^{0}(M)\right)=\operatorname{Deg}(M) .
\end{aligned}
$$

Now we are touching upon the theory of Gröbner bases where we assume that the field $K$ is infinite. Let $F=\bigoplus_{i=0}^{r} R e_{i}$ be a free graded $R$-module of rank $r$. We will always use the reverse lexicographic order on the monomials of $F$, i.e. if $m, n \in R$ are monomials, then $m e_{i}>n e_{j}$ if either $\operatorname{deg} m e_{i}>\operatorname{deg} n e_{j}$ or the degrees are the same and $m>n$ in the reverse lexicographic order of the monomials in $R$ or $m=n$ and $i<j$.

Denote by GL $(n+1)$ the group of $K$-linear graded automorphisms of $R$ and let $\mathrm{GL}(F)$ be the group of $R$-linear graded automorphisms of $F$. Then $G:=\mathrm{GL}(n+1) \ltimes$ GL $(F)$ acts on $F$ through $K$-linear graded automorphisms. Let $B$ be the subgroup of $G$ consisting of all automorphisms that take $e_{i}$ to an $R$-linear combination of $e_{1}, \ldots, e_{i}$ and $x_{i}$ to a $K$-linear combination of $x_{0}, \ldots, x_{i}$. It is a Borel group of 
G. A submodule $M$ of $F$ is called Borel-fixed if $\gamma(M)=M$ for all $\gamma \in B$. A combinatorial characterization of such submodules is given in [12, Proposition II.5. They occur naturally because of the following result. For every graded submodule $M$ of $F$ there is a Zariski open subset $U \subset G$ and a Borel-fixed module $N$ such that $N=\operatorname{in}(\gamma(M))$ for all $\gamma \in U$ ([12, Example I.7, or modify the proofs in [6] or [7] for the case $F=R$ ). The module $N$ is called the generic initial module of $M$ and is denoted by $\operatorname{gin}(M)$.

Generic initial modules can be used to compute the smallest extended degree. This result extends Theorem 3.4.2 in [8].

Lemma 4.3. For every graded submodule $M$ of $F$ we have

$$
\operatorname{bdeg}(F / M)=\operatorname{bdeg}(F / \operatorname{gin}(M)) .
$$

Proof. The proof is analogous to the one of Gunston for the case $F=R$. We sketch it for the sake of completeness.

We induct on $\operatorname{dim} F / M$. The 0 -dimensional case being clear we may assume $\operatorname{dim} F / M \geq 1$. First, suppose that $\operatorname{depth} F / M>0$. Let $l \in R$ be a general linear form. Then we get by induction

$$
\begin{aligned}
\operatorname{bdeg}(F / M) & =\operatorname{bdeg}(F / M+l F)=\operatorname{bdeg}(F / \operatorname{gin}(M+l F)) \\
& =\operatorname{bdeg}\left(F / \operatorname{gin}(M)+x_{n} F\right)=\operatorname{bdeg}(F / \operatorname{gin}(M)+l F) \\
& =\operatorname{bdeg}(F / \operatorname{gin}(M)),
\end{aligned}
$$

where we have also used properties of generic initial modules under hyperplane sections that are straightforward extensions of Green's results [7] for generic initial ideals.

Second, assume $\operatorname{depth} F / M=0$. We will use the saturation of $M$, i.e.

$$
M^{\text {sat }}:=\bigcup_{k \geq 1}\left(M:_{F} \mathfrak{m}^{k}\right) .
$$

Since depth $F / M^{\text {sat }}>0$ and saturation commutes with formation of generic initial modules we obtain

$$
\begin{aligned}
\operatorname{bdeg}(F / M) & =\operatorname{bdeg}\left(F / M^{\text {sat }}\right)+\ell\left(M^{\text {sat }} / M\right) \\
& =\operatorname{bdeg}\left(F / \operatorname{gin}(M)^{\text {sat }}\right)+\ell\left(\operatorname{gin}(M)^{\text {sat }} / \operatorname{gin}(M)\right) \\
& =\operatorname{bdeg}(F / \operatorname{gin}(M))
\end{aligned}
$$

and we are done.

We are ready for the main results of this section. The first one shows that the converse of Theorem 3.5 is true if we use the smallest extended degree. It also gives a nice tie with the theory of Gröbner bases.

Theorem 4.4. Let $I$ be a homogeneous ideal in $R=K\left[x_{0}, \ldots, x_{n}\right]$ where $K$ is an infinite field. Assume that I does not contain any linear form. Then the following conditions are equivalent:

(a) $\operatorname{reg}(R / I)=\operatorname{bdeg}(R / I)-1$ and $\operatorname{depth} R / I=t$.

(b) $I$ is an ideal as in Lemma 3.4 with $n+1-t$ minimal generators.

(c) There are integers $d_{t}, \ldots, d_{n} \geq 0$ with $0 \leq t \leq n, d_{n}, d_{t}>0$ such that

$$
\operatorname{gin}(I)=\left(x_{0}^{d_{n}+1}, x_{0}^{d_{n}} x_{1}^{d_{n-1}+1}, \ldots, x_{0}^{d_{n}} \ldots x_{n-t-2}^{d_{t+2}} x_{n-t-1}^{d_{t+1}+1}, x_{0}^{d_{n}} \ldots x_{n-t-1}^{d_{t+1}} x_{n-t}^{d_{t}}\right) .
$$


Proof. Theorem 3.5 shows that (a) implies (b). In order to prove the converse we have to show for every ideal $I$ as in (b) that

$$
\operatorname{bdeg}(R / I)=d_{t}+\ldots+d_{n}
$$

because $\operatorname{reg}(R / I)=d_{t}+\ldots+d_{n}-1$ as a consequence of Lemma 3.4 .

We will induct on $n$. Condition (1) for bdeg and Lemma 3.4 provide

$$
\begin{aligned}
\operatorname{bdeg}(R / I) & =\operatorname{bdeg}\left(R / I^{\text {sat }}\right)+\ell\left(H_{\mathfrak{m}}^{0}(R / I)\right) \\
& =\operatorname{bdeg}\left(R / I^{\text {sat }}\right)+d_{0} .
\end{aligned}
$$

In particular, this implies the claim for $n=0$, thus we may assume $n \geq 1$. Let $s=\min \left\{i \geq 1 \mid d_{i} \geq 1\right\}$. This minimum exists because $d_{n} \geq 1$. Then, using Lemma 3.4 we see that

$$
I^{s a t}=\left(f_{n} l_{0}, f_{n} f_{n-1} l_{1}, \ldots, f_{n} \ldots f_{s+1} l_{n-s-1}, f_{n} \ldots f_{s}\right)
$$

is again an ideal as in Lemma 3.4 where $\operatorname{depth} R / I^{\text {sat }}=s$. Hence, it remains to consider the case where $t=\operatorname{depth} R / I>0$. Changing coordinates we may assume that $x_{n}$ is general for $R / I$ and we have

$$
\operatorname{bdeg}(R / I)=\operatorname{bdeg}\left(R / I+x_{n} R\right) .
$$

But $R / I+x_{n} R \cong \bar{R} / \bar{I}$ with $\bar{R}=R / x_{n} R$ and

$$
\bar{I}=\left(g_{n-1} \bar{l}_{0}, g_{n-1} g_{n-2} \bar{l}_{1}, \ldots, g_{n-1} \ldots g_{t} \bar{l}_{n-t-1}, g_{n-1} \ldots g_{t-1}\right)
$$

where $g_{i}$ is the residue class of $f_{i+1}$ and $\bar{l}_{i}$ is the residue class of $l_{i}$. We may apply the induction hypothesis to $\bar{I}$. Hence we obtain

$$
\operatorname{bdeg}(\bar{R} / \bar{I})=d_{t}+\ldots+d_{n}
$$

which provides $\operatorname{bdeg}(R / I)=d_{t}+\ldots+d_{n}$, as claimed.

Now, we show that (c) implies (a). Note that the given $\operatorname{gin}(I)$ is an ideal as in Lemma 3.4. Hence, we have just seen that $t=\operatorname{depth} R / \operatorname{gin}(I)$ and

$$
\operatorname{reg}(R / \operatorname{gin}(I))=\operatorname{bdeg}(R / \operatorname{gin}(I))-1 .
$$

According to [3] we have

$$
\operatorname{reg}(R / I)=\operatorname{reg}(R / \operatorname{gin}(I)) .
$$

Combined with Lemma 4.3 and $\operatorname{depth} R / I=\operatorname{depth} R / \operatorname{gin}(I)$ we get the equations in (a).

Reversing the last argument we see that (a) implies the fact that $\operatorname{gin}(I)$ is an ideal as in Lemma 3.4 and $\operatorname{depth} R / \operatorname{gin}(I)=t$. Since $\operatorname{gin}(I)$ is a Borel-fixed ideal it must be of the form as claimed.

Remark 4.5. Unless the ideal is principal the ideals occurring in Theorem 4.4(b) are not unmixed. However, the lower-dimensional components are not necessarily embedded.

Combined with Lemma 3.4. Theorem 4.4 says precisely for which Hilbert series $H$ where is a graded $K$-algebra $A$ with $H_{A}=H$ and $\operatorname{reg}(A)=\operatorname{bdeg}(A)-1$. A lot more is true. There is another purely numerical characterization of these algebras. 
Theorem 4.6. Let $A \neq K$ be a standard graded $K$-algebra where $K$ is an infinite field. Then the following conditions are equivalent:

(a) A has maximal regularity with respect to the smallest extended degree, i.e.

$$
\operatorname{reg}(A)=\operatorname{bdeg}(A)-1 .
$$

(b) The Hilbert series of $A$ is

$$
H_{A}(z)=\sum_{j=0}^{n} \frac{1-z^{d_{j}}}{(1-z)^{j+1}} \prod_{i=j+1}^{n} z^{d_{i}}
$$

for some integers $d_{0}, \ldots, d_{n} \geq 0$ where $n:=\operatorname{dim} A$ and $d_{n}>0$.

Remark 4.7. Using Theorem 4.4 we see that the integers $d_{i}$ occurring in condition (b) have a cohomological interpretation. Indeed, Lemma 3.4 shows that $H_{\mathfrak{m}}^{i}(A)^{\vee}$ is (up to a degree shift) a hypersurface ring of degree $d_{i}$. Here degree zero means that the cohomology module is trivial.

Proof of Theorem 4.6. One direction is clear. Theorem 4.4 in conjunction with Lemma 3.4 shows that (a) implies (b).

For the other implication we prove by induction on $n$ that (b) has as a consequence

$$
\operatorname{reg}(A)=\operatorname{bdeg}(A)-1=d_{0}+\ldots+d_{n}-1 .
$$

This is clear if $n=0$. Let $n \geq 1$. Notice that the Hilbert series of $A$ conveys that $d_{n}=\operatorname{deg} A$ and $n+1=h_{A}(1)=\operatorname{rank}_{K}[A]_{1}$. Thus, we see that $A \cong R / I$ where $R=K\left[x_{0}, \ldots, x_{n}\right]$. Since $\operatorname{dim} A=n$ the ideal $I$ has codimension one in $R$. Hence, the greatest common divisor of the elements in $I$ is a homogeneous polynomial $f$ of degree $d_{n}=\operatorname{deg} A$. Setting $B:=R / I: f$, multiplication by $f$ on $A$ provides the exact sequence

$$
0 \rightarrow B\left(-d_{n}\right) \rightarrow A \rightarrow R / f R \rightarrow 0 .
$$

Hence the Hilbert series of $B$ is

$$
H_{B}(z)=\sum_{j=0}^{n-1} \frac{1-z^{d_{j}}}{(1-z)^{j+1}} \prod_{i=j+1}^{n-1} z^{d_{i}}
$$

and the induction hypothesis provides

$$
\operatorname{reg}(B)=\operatorname{bdeg}(B)-1=d_{0}+\ldots+d_{n-1}-1 .
$$

Since $\operatorname{reg}(R / f R)=d_{n}-1$ we obtain from the sequence (4.1)

$$
\operatorname{reg}(A)=\operatorname{reg}(B)+d_{n}=d_{0}+\ldots+d_{n}-1 .
$$

It remains to compute $\operatorname{bdeg}(A)$. Due to Theorem 4.4, Lemma 3.4 applies to $B$ and shows that the Hilbert series of $H_{\mathfrak{m}}^{0}(B)$ is

$$
H_{H_{\mathfrak{m}}^{0}(B)}(z)=\frac{1-z^{d_{0}}}{1-z} \prod_{i=1}^{n} z^{d_{i}} .
$$

Using the sequence (4.1) again we get that $H_{\mathfrak{m}}^{0}(A) \cong H_{\mathfrak{m}}^{0}(B)$. Therefore the Hilbert series of $C:=A / H_{\mathrm{m}}^{0}(A)$ is

$$
\begin{aligned}
H_{C}(z) & =H_{A}(z)-H_{H_{\mathfrak{m}}^{0}(B)}(z) \\
& =\sum_{j=1}^{n} \frac{1-z^{d_{j}}}{(1-z)^{j+1}} \prod_{i=j+1}^{n} z^{d_{i}} .
\end{aligned}
$$


Let $l \in R$ be a general linear form. It is not a zero divisor on $C$. Hence we get for the Hilbert series of $C / l C$

$$
H_{C / l C}(z)=(1-z) \cdot H_{C}(z)=\sum_{j=1}^{n} \frac{1-z^{d_{j}}}{(1-z)^{j}} \prod_{i=j+1}^{n} z^{d_{i}} .
$$

Thus, we can apply the induction hypothesis to $C / l C$ and obtain

$$
\operatorname{bdeg}(C)=\operatorname{bdeg}(C / l C)=d_{1}+\ldots+d_{n} .
$$

Since $H_{\mathfrak{m}}^{0}(A)$ has length $d_{0}$ it follows that

$$
\operatorname{bdeg}(A)=\operatorname{bdeg}(C)+\ell\left(H_{\mathfrak{m}}^{0}(A)\right)=d_{0}+\ldots+d_{n},
$$

completing the proof.

Expressing the information on the Hilbert series of the algebras more explicitly by using the Hilbert function illustrates again how special these algebras are. We work this out in low dimensions.

Example 4.8. Let $A$ be an algebra as in Theorem 4.6(b).

(i) If $A$ is artinian, then the Hilbert function of $A$ is $1,1, \ldots, 1,0,0 \ldots$

(ii) If $\operatorname{dim} A=1$, then the Hilbert function is

$$
h_{A}(j)=\left\{\begin{array}{cl}
j+1 & \text { if } 0 \leq j \leq d_{1}-1, \\
d_{1}+1 & \text { if } d_{1} \leq j \leq d_{0}+d_{1}-1, \\
d_{1} & \text { if } d_{0}+d_{1} \leq j .
\end{array}\right.
$$

Using Lemma 3.3 it is possible to extend the previous results to modules. For example, the module version of Theorem 4.6 is.

Corollary 4.9. Let $R$ be a noetherian polynomial ring over the infinite field $K$, and let $M \neq 0$ be a finitely generated graded $R$-module. Then the following conditions are equivalent:

(a) $M$ has maximal regularity with respect to the smallest extended degree, i.e.

$$
\operatorname{reg}(M)=e_{R}^{+}(M)+\operatorname{bdeg}(M)-1 .
$$

(b) $M$ is a cyclic R-module with Hilbert series

$$
H_{M}(z)=z^{k} \cdot\left[\sum_{j=0}^{n} \frac{1-z^{d_{j}}}{(1-z)^{j+1}} \prod_{i=j+1}^{n} z^{d_{i}}\right]
$$

for some integers $k, d_{0}, \ldots, d_{n}$, where $n=\operatorname{dim} M, d_{0}, \ldots, d_{n-1} \geq 0$, and $d_{n}>0$.

Proof. Using $H_{M(-k)}(z)=z^{k} \cdot H_{M}(z)$ this follows by combining Lemma 3.3 and Theorem 4.6.

\section{Comments And open Problems}

We begin by discussing the assumption about the ground field that we have made in the previous sections. Then we show that our results broadly generalize the main result of Stückrad and Vogel in [14. 
Remark 5.1. The assumption that the ground field $K$ is infinite was necessary in order to guarantee that there are filter regular elements. However, typically one would expect that a degree does not change when the base field $K$ is extended. If we add this requirement as an additional condition for an extended degree, then Theorem 3.1 and Corollary 3.2 become valid over arbitrary ground fields. Indeed, we could replace $K$ by its algebraic closure if necessary. Note that Vasconcelos' homological degree satisfies the extra requirement above.

Every extended degree Deg satisfies $\operatorname{Deg}(M) \geq \operatorname{deg}(M)$ and equality holds if and only if $M$ is a Cohen-Macaulay module. Following [5], the difference

$$
I(M):=\operatorname{Deg}(M)-\operatorname{deg}(M)
$$

is called the Cohen-Macaulay deviation of $M$. Using it we can state another consequence of Theorem 2.5

Proposition 5.2. Let $M$ be a finitely generated graded $R$-module of dimension $d \geq 1$. Let $\mathfrak{q} \subset R$ be a parameter ideal generated by $d$ sufficiently general linear forms. Suppose that the ground field $K$ is infinite. Then we have for any extended degree

$$
\ell(M / \mathfrak{q} M)-e_{0}(\mathfrak{q} ; M)-\operatorname{reg}(M / \mathfrak{q} M) \leq I(M)-\operatorname{reg}(M),
$$

where $e_{0}(\mathfrak{q} ; M)$ denotes the multiplicity of $\mathfrak{q}$ (cf., e.g., [17], Chapter VIII).

Proof. Successive application of Theorem 2.5 provides

$$
\begin{aligned}
\ell(M / \mathfrak{q} M)-\operatorname{reg}(M / \mathfrak{q} M) & \leq \operatorname{Deg}(M)-\operatorname{reg}(M) \\
& =\operatorname{deg}(M)+I(M)-\operatorname{reg}(M) .
\end{aligned}
$$

Since the minimal generators of $\mathfrak{q}$ are sufficiently general by assumption, they form an $M$-filter regular sequence. Thus, [2, Proposition 4.7, shows that they form a reducing system of parameters for $M$. Hence, we have $e_{0}(\mathfrak{q} ; M)=\operatorname{deg}(M)$. Combined with the estimate above the claim follows.

It is useful to specify what means "sufficiently general" for the linear forms in the last result provided we deal with a specific extended degree. All we need is to know which assumptions on the linear form $l$ guarantee that $\operatorname{Deg}(M / l M) \leq \operatorname{Deg}(M)$. This follows by an analysis of the proof of Theorem 2.5

In case of the homological degree this has been achieved by Vasconcelos. Following [16], Definition 2.12, a linear form $l \in R$ is called a special hyperplane section of $M$ if it is filter regular for all the iterated extension modules

$$
\operatorname{Ext}_{R}^{i_{1}}\left(\operatorname{Ext}_{R}^{i_{2}}\left(\ldots\left(\operatorname{Ext}_{R}^{i_{p}}(M, R), R\right), \ldots, R\right)\right)
$$

where $i_{1} \geq i_{2} \geq \ldots \geq i_{p} \geq 0$ are any integers.

Suppose that the module $M$ has dimension $d$. We call the ideal $\mathfrak{q}=\left(l_{1}, \ldots, l_{d}\right) \subset$ $R$ a special parameter ideal of $M$ if $l_{i}$ is a special hyperplane section of $M /\left(l_{1}, \ldots, l_{i-1}\right) M$ for all $i=1, \ldots, d$.

In case of the homological degree we denote the Cohen-Macaulay deviation of the module $M$ by

$$
I_{h}(M)=\operatorname{hdeg}(M)-\operatorname{deg}(M) .
$$


Using this notation Proposition 5.2 provides:

Corollary 5.3. Let $\mathfrak{q} \subset R$ be a special parameter ideal of the module $M$. Then we have for the homological degree

$$
\ell(M / \mathfrak{q} M)-e_{0}(\mathfrak{q} ; M)-\operatorname{reg}(M / \mathfrak{q} M) \leq I_{h}(M)-\operatorname{reg}(M) .
$$

Proof. Vasconcelos' Theorem 2.13 in [16] shows for a special hyperplane section $l$ of $M$ that

$$
\operatorname{hdeg}(M / l M) \leq \operatorname{hdeg}(M) .
$$

As pointed out above, the claim then follows by Proposition 5.2 .

The last result can be further simplified if $M$ is a module with finite cohomology, i.e. if all the modules $H_{\mathfrak{m}}^{i}(M), 0 \leq i<\operatorname{dim} M$, have finite length. Note that this is the case if and only if $M$ is an equidimensional locally Cohen-Macaulay module. It is easy to see that then a linear form is a special hyperplane section of the module $M$ if and only if it is a parameter for $M$. Thus, the last corollary implies:

Corollary 5.4. Let $M$ be a an equidimensional locally Cohen-Macaulay graded module. Let $\mathfrak{q} \subset R$ be an ideal generated by a system of linear parameters of $M$ and suppose that $K$ is infinite. Then we have

$$
\ell(M / \mathfrak{q} M)-e_{0}(\mathfrak{q} ; M)-\operatorname{reg}(M / \mathfrak{q} M) \leq I_{h}(M)-\operatorname{reg}(M) .
$$

Note that with the assumptions of the last result, the Cohen-Macaulay deviation is

$$
I_{h}(M)=\sum_{0}^{d-1}\left(\begin{array}{c}
d-1 \\
i
\end{array}\right) \ell\left(H_{\mathfrak{m}}^{i}(M)\right),
$$

where $d=\operatorname{dim} M$. Hence, Corollary 5.4 is a generalization of the Main theorem in [14] (cf. also [1], [10]) from algebras to modules. There Stückrad and Vogel have also considered the problem for which algebras we have equality in Corollary 5.4 for every ideal $\mathfrak{q}$ under consideration. Using Corollary 2.6 their methods can be extended to the case of modules and provide, for example, the following result. If $M$ has positive depth, then we have equality in Corollary 5.4 for every ideal $\mathfrak{q}$ under consideration if and only if $M$ is a Buchsbaum module.

It seems interesting to analyze the analogous problem in the more general situation of Corollary 5.3. We pose this as an open problem.

\section{ACKNOWLEDGEMENT}

The author thanks W. Vasconcelos for helpful comments.

\section{REFERENCES}

[1] R. Achilles, P. Schenzel, On bounds for Castelnuovo's index of regularity, J. Math. Kyoto Univ. 29 (1989), 91-104. MR90i:14002

[2] M. Auslander, D. A. Buchsbaum, Codimension and multiplicity, Ann. Math. 68 (1958), 625-657. MR20:6414

[3] D. Bayer, M. Stillman, A criterion for detecting m-regularity, Invent. Math. 87 (1987), 1-11. MR $87 \mathrm{k}: 13019$

[4] M. Brodmann, U. Nagel, Bounding cohomological Hilbert functions by hyperplane sections, J. Algebra 174 (1995), 323-348. MR96d:14017

[5] L. R. Doering, T. Gunston, W. V. Vasconcelos, Cohomological degrees and Hilbert functions of graded modules, Amer. J. Math. 120 (1998), 493-504. MR99h:13019 
[6] D. Eisenbud, "Commutative algebra with a view toward algebraic geometry," Graduate Texts in Mathematics 150, Springer-Verlag, 1995. MR97a:13001

[7] M. Green, Generic initial ideals, In: "Six lectures on commmutative algebra" (J. Elias, J. M. Giral, R. M. Miro-Roig, S. Zarzuela, Eds.), Progress in Mathematics 166, Birkhäuser, 1998, pp. 119-185. MR.99m:13040

[8] T. Gunston, Cohomological degrees, Dilworth numbers and linear resolution, Ph.D. Thesis, Rutgers University, 1998.

[9] J. Migliore, U. Nagel, C. Peterson, Bezout's theorem and Cohen-Macaulay modules, Math. Z. 237 (2001), 373-394. MR2002e:13030

[10] U. Nagel, Castelnuovo-Regularität und Hilbertreihen, Math. Nachr. 142 (1989), 27-43. MR $90 \mathrm{~m}: 13025$

[11] U. Nagel, On Castelnuovo's regularity and Hilbert functions, Compositio Math. 76 (1990), 265-275. MR92c:14003

[12] K. Pardue, Nonstandard Borel-fixed ideals, Ph.D. Thesis, Brandeis University, 1994.

[13] M. E. Rossi, N. V. Trung, G. Valla, Castelnuovo-Mumford regularity and extended degree, Trans. Amer. Math. Soc. 355 (2003), 1773-1786. MR2004b:13020

[14] J. Stückrad, W. Vogel, Castelnuovo's regularity and multiplicity, Math. Ann. 281 (1988), 355-368. MR90b:14059a

[15] W. V. Vasconcelos, Cohomological degrees of graded modules, In: "Six lectures on commutative algebra" (J. Elias, J. M. Giral, R. M. Miro-Roig, S. Zarzuela, Eds.), Progress in Mathematics 166, Birkhäuser, Basel, 1998, pp. 345-392. MR99j:13012

[16] W. V. Vasconcelos, The homological degree of a module, Trans. Amer. Math. Soc. 350 (1998), 1167-1179. MR98i:13046

[17] O. Zarisky, P. Samuel, "Commutative algebra," Vol. II, van Nostrand, Toronto, New York, London, 1960. MR 22:11006

Department of Mathematics, University of Kentucky, 715 Patterson Office Tower, LeXington, KentuCKy 40506-0027

E-mail address: uwenagel@ms.uky.edu 